\title{
Identification of Tropical Plant Extracts That Extend Yeast Chronological Life Span
}

\author{
Mandy Mun Yee Kwong ${ }^{1,2}$, Jee Whu Lee ${ }^{1,2}$, Mohammed Razip Samian ${ }^{2,3}$, Habibah A. Wahab 2,4(1), \\ Nobumoto Watanabe ${ }^{2,5}$ and Eugene Boon Beng Ong 1,2,*(D) \\ 1 Institute for Research in Molecular Medicine (INFORMM), Universiti Sains Malaysia-USM, \\ Penang 11800, Malaysia; libragirl1015@gmail.com (M.M.Y.K.); jeewhu@student.usm.my (J.W.L.) \\ 2 USM-RIKEN International Centre for Ageing Science (URICAS), Universiti Sains Malaysia-USM, \\ Penang 11800, Malaysia; razip.samian@gmail.com (M.R.S.); habibahw@usm.my (H.A.W.); \\ nwatanab@riken.jp (N.W.) \\ 3 School of Biological Sciences, Universiti Sains Malaysia-USM, Penang 11800, Malaysia \\ 4 School of Pharmaceutical Sciences, Universiti Sains Malaysia-USM, Penang 11800, Malaysia \\ 5 Bio-Active Compounds Discovery Research Unit, RIKEN Center for Sustainable Resource Science, \\ Saitama 351-0198, Japan \\ * Correspondence: eugene@usm.my
}

check for updates

Citation: Kwong, M.M.Y.; Lee, J.W.; Samian, M.R.; Wahab, H.A.; Watanabe, N.; Ong, E.B.B.

Identification of Tropical Plant

Extracts That Extend Yeast Chronological Life Span. Cells 2021, 10, 2718. https://doi.org/10.3390/ cells10102718

Academic Editors: Milena Georgieva and Mateusz Mołoń

Received: 27 August 2021

Accepted: 8 October 2021

Published: 11 October 2021

Publisher's Note: MDPI stays neutral with regard to jurisdictional claims in published maps and institutional affiliations.

Copyright: (c) 2021 by the authors. Licensee MDPI, Basel, Switzerland. This article is an open access article distributed under the terms and conditions of the Creative Commons Attribution (CC BY) license (https:// creativecommons.org/licenses/by/ $4.0 /)$.

\begin{abstract}
Certain plant extracts (PEs) contain bioactive compounds that have antioxidant and lifespan-extending activities on organisms. These PEs play different roles in cellular processes, such as enhancing stress resistance and modulating longevity-defined signaling pathways that contribute to longevity. Here, we report the discovery of PEs that extended chronological life span (CLS) in budding yeast from a screen of 222 PEs. We identified two PEs, the leaf extracts of Manihot esculenta and Wodyetia bifurcata that extended CLS in a dose-dependent manner. The CLS-extending PEs also conferred oxidative stress tolerance, suggesting that these PEs might extend yeast CLS through the upregulation of stress response pathways.
\end{abstract}

Keywords: ageing; life span; plant extracts; stress response; Saccharomyces cerevisiae

\section{Introduction}

Ageing is a biological process that occurs with gradual structural and functional changes [1,2] in all living organisms that eventually leads to death [3]. With the passage of time, cellular and body systems start to deteriorate with the accumulation of cellular damages and toxic substances in cells [4]. Various studies on ageing have been performed with different model organisms such as Mus musculus (mouse), Caenorhabditis elegans (worm), Drosophila melanogaster (fruit fly), and Saccharomyces cerevisiae (budding yeast). Model organisms have a relatively shorter generation time and life spans that range from weeks to months, and their smaller sizes are advantageous for laboratory high throughput ageing experiments. Additionally, unicellular organisms such as yeast allow us to study the molecular mechanisms of ageing and senescence at the cellular level [5].

Cellular chronological ageing is a type of ageing that occurs in postmitotic, nonreplicating cells. In yeast, chronological life span (CLS) is defined as the length of time that a non-dividing yeast cell is viable and can re-enter the cell cycle to reproduce new cells upon the introduction of a new growth medium [6-8]. CLS assay of non-dividing yeast cells is a model that resembles the ageing process in postmitotic cells such as neurons and muscle cells $[9,10]$, thus the yeast model can provide useful insights into cellular ageing.

Natural phytochemicals found in various plant extracts (PEs) were shown to have pharmacological value towards living organisms and contribute to life span extension in model organisms without affecting the essential biological functions of cells [11-13]. It is widely thought that the antioxidant activity of the phytochemicals is one of the main contributors to anti-ageing [14]. For example, it was reported that the antioxidant activities 
of aqueous polyphenols such as tannic acid and glucosides in Rosa rugosa [15], and the carotenoids, chlorophylls and flavonoids in the fruit pulp of Dipteryx alata [16,17] contribute to the life span extension of $C$. elegans.

Several screenings of PEs were performed to study their effects on CLS and their roles in ageing-related cellular processes and mechanisms in budding yeast S. cerevisiae. For instance, a screen of 53 PEs discovered 15 extracts, respectively, from berries of Serenoa repens, aerial parts of Hypericum perforatum, leaves of Ilex paraguariensis, leaves of Ocimum tenuiflorum, whole plant of Solidago virgaurea, fruits of Citrus sinensis, whole plant of Humulus lupulus, grape skins of Vitis vinifera, whole plant of Andrographis paniculata, roots of Hydrastis canadensis, seeds of Trigonella foenum-graecum, root barks of Berberis vulgaris leaves, flowers and stems of Crataegus monogyna, leaves of Taraxacum erythrospermum, and the whole plant of Ilex paraguariensis that prolong yeast CLS. These 15 PEs were shown to play roles in cellular processes such as enhancing mitochondrial respiration, reducing cellular reactive oxygen species (ROS) level, protecting cellular genetics and proteins from oxidative damage and promoting oxidative and thermal stress tolerance for extending CLS [18]. In addition, a screen of 35 PEs identified six extracts, respectively, from root and rhizome of Cimicifuga racemosa, root of Valeriana officinalis L., whole plant of Passiflora incarnate L., leaf of Ginkgo biloba, seed of Apium graveolens L., and bark of Salix alba that increase yeast CLS via different involvement in the longevity-defined cellular processes as described formerly for the 15 PEs [19]. Moreover, these six CLS-extending PEs are also involved differently in highly conserved longevity-regulating signalling pathways such as target of rapamycin complex 1 (TORC1) pathway [20,21], protein kinase A (PKA) pathway [21], and Pkb-activating kinase homolog (PKH1/2) pathway [22], which prolong ageing, and sucrose non-fermenting (SNF1) pathway [23] and autophagy (ATG) pathway [24,25], which delay ageing [26].

In this study, we sought to identify tropical plants extracts that can extend yeast CLS. As a preliminary step towards the discovery of phytochemicals that may regulate ageing, we used a 96-well plate-based CLS outgrowth assay to screen 222 crude methanolic PEs from 175 species of 70 plant families. We initially identified seven candidate PEs that extended yeast CLS and further characterised the top three potential PEs in terms of their dose-dependent activity and stress tolerance during chronological ageing. We show that two of the PEs have dose-dependent effects and that the PEs potentially confer oxidative stress tolerance during chronological ageing. Our findings reveal the presence of ageing regulating molecules in non-herbal or medicinal plants and highlight the case for continuous bioprospecting from tropical plants.

\section{Materials and Methods}

\subsection{Yeast Strains, Media and Growth Conditions}

The S. cerevisiae strain 1783 (MATa can1 his4 leu2-3,112 trp1-1 ura3-52) (National BioResource Project, Hiroshima, Japan) was used in this study. Yeast cells were cultured at $30{ }^{\circ} \mathrm{C}$ with shaking at $200 \mathrm{rpm}$ in Yeast Peptone Dextrose (YPD) medium $(10 \mathrm{~g} / \mathrm{L}$ yeast extract, $20 \mathrm{~g} / \mathrm{L}$ peptone, and $20 \mathrm{~g} / \mathrm{L}$ dextrose) (HIMEDIA, Mumbai, India) for general growth. Synthetic complete (SC) medium consisting of $2 \%(w / v)$ glucose (HIMEDIA, Mumbai, India), $0.67 \%(w / v)$ yeast nitrogen base with ammonium sulphate (HIMEDIA, Mumbai, India), complete supplement mixture without tryptophan (HIMEDIA, Mumbai, India) and supplemented tryptophan (HIMEDIA, Mumbai, India) was used for ageing cultures. The concentrations of amino acids in SC medium are listed in Supplementary Table S1.

\subsection{Preparation of Plant Extract (PE) Library}

A total of 222 crude methanolic PEs derived from 175 species from 70 families of plants was obtained from the USM-RIKEN Joint Laboratory for Bioprobe Discovery. A total of 20 milligrams of PEs were dissolved in $1 \mathrm{~mL}$ of $100 \%$ dimethyl sulfoxide (DMSO) to a final concentration of $20 \mathrm{mg} / \mathrm{mL}$ in $1.5 \mathrm{~mL}$ microcentrifuge tubes, respectively, and stored at $-20^{\circ} \mathrm{C}$. The working stocks of PEs were prepared at $10 \mathrm{mg} / \mathrm{mL}$ by diluting $10 \mu \mathrm{L}$ 
of $20 \mathrm{mg} / \mathrm{mL}$ PEs each with $10 \mu \mathrm{L}$ of DMSO in 96-well microplates. The microplate plates were sealed with adhesive film and stored upright at $-20^{\circ} \mathrm{C}$. All the PEs were labelled with a code that matches their locations on the 96-well plate. A PE library with information of each PE (local name, scientific name, family name, plant organ, and PE code) is listed (Supplementary Table S2).

\subsection{Chronological Life Span Assay}

The CLS assay for screening PEs was based on a 96-well plate CLS assay we had established previously [27]. First, the yeast strain 1783 was streaked on YPD agar and incubated for 2 days. A single colony was selected and grown overnight in a Bijou bottle with $1 \mathrm{~mL}$ of YPD medium at $30{ }^{\circ} \mathrm{C}$ with shaking at $200 \mathrm{rpm}$. A total of 3 colonies were selected as 3 biological replicates of PE-treated culture or control culture. An amount of $1.8 \mu \mathrm{L}$ overnight culture was transferred to each well of 96-well flat-bottom microplate (IWAKI) containing $176.4 \mu \mathrm{L}$ of SC medium and $1.8 \mu \mathrm{L}$ of PEs at final concentration of $100 \mu \mathrm{g} / \mathrm{mL}$. Culture of cells grown in SC medium with $1.8 \mu \mathrm{L}$ of DMSO served as the control. The mixtures were suspended several times with multichannel pipette to ensure even distribution of PEs in the liquid culture. The cultures in the microplate were incubated at $30{ }^{\circ} \mathrm{C}$ without agitation throughout the 8 days of ageing. The day after an initial $48 \mathrm{~h}$ of growth was defined as day 0 of the stationary phase. On day $0,2,4$, and 6 of the stationary phase, an aliquot of $2 \mu \mathrm{L}$ of each replicate of ageing culture was transferred to a new 96-well flat bottom microplate (IWAKI) containing $98 \mu \mathrm{L}$ YPD medium to screen the PEs. After transferring the aliquots of cultures to a new microplate, the aged cultures were re-incubated at $30{ }^{\circ} \mathrm{C}$.

In the primary screen, the outgrowths of yeast cultures in the new 96-well microplate were monitored by measuring the absorbance at a wavelength of $600 \mathrm{~nm}\left(\mathrm{~A}_{600}\right)$ every $6 \mathrm{~h}$ until $24 \mathrm{~h}$ using Bio Microplate Reader HiTS (Cosmo Bio, Tokyo, Japan). From the primary screen, the PEs that increased the relative absorbance of culture (average absorbance value of each PE-treated culture at the 12th h divided by average absorbance value of the control culture at 12th $\mathrm{h}$ ), which was higher than that of the control culture throughout the ageing period (Supplementary Tables S3-S6, Figure S6), were selected for the secondary screen.

In the secondary and confirmatory screens, the outgrowths of yeast cultures in the new 96-well microplate were monitored by measuring the absorbance $\left(\mathrm{A}_{600}\right)$ every $30 \mathrm{~min}$ until 24 h using Bio Microplate Reader HiTS (Cosmo Bio, Tokyo, Japan). The cultures in the 96-well microplate were incubated at $30{ }^{\circ} \mathrm{C}$, with continuous shaking at $210 \mathrm{rpm}$ in the reader [27]. The PEs that increased the survival percentage of cultures throughout the ageing period in the secondary screen (Supplementary Figure S9) were selected for the confirmatory screen. Survival percentage of each replicate of aged culture was determined by the formula: $S_{n}=\frac{1}{2\left(\frac{\Delta t_{n}}{\delta_{r}}\right)} \times 100 \%$, whereby $S_{n}=$ survival percentage on day $n, \Delta t_{n}=$ time shift from day 0 to day $\mathrm{n}$ at $\mathrm{A}_{600} 0.3$, determined from the linear regression equation of natural logarithm of $A_{600}$ and $\delta_{r}=$ doubling time of each replicate. This $\delta_{\mathrm{r}}$ was calculated from the maximal slope of the semilog plot of absorbance against time and was defined as the average of at least three $\delta$ values for that replicate. Doubling times $(\delta)$ were calculated between every consecutive pair of absorbance values between 0.2 and 0.5 , by the formula: $\delta=\frac{\ln (2)}{\left(\frac{\ln \left(A_{2}\right)-\ln \left(A_{1}\right)}{t_{2}-t_{1}}\right)}$, whereby $A_{2}$ and $A_{1}$ are successive absorbance values while $t_{1}$ and $t_{2}$ are the times of consecutive pairs of respective absorbance values. The survival percentage of each replicate of each PE-treated culture was then averaged. A graph of average survival percentage against ageing period (day) was plotted [6]. From the survival percentage graph, survival integral (SI) was calculated as the area under the curve (AUC) by applying mathematical trapezium rule: $\mathrm{SI}=\sum_{2}^{n}\left(\frac{S_{n-2}+S_{n}}{2}\right)\left(\right.$ day $_{\mathrm{n}}-$ day $\left._{\mathrm{n}-2}\right)$, whereby dayn is the day of outgrowth measurement (day Identification of tropical plant extracts that extend yeast chronological lifespan 2, 4, 6 in this study) [6]. 


\subsection{Dose-Dependent Effects of Plant Extracts on Yeast Chronological Life Span}

A single colony of yeast strain 1783 was selected from YPD agar and grown overnight in a Bijou bottle with $1 \mathrm{~mL}$ of YPD medium at $30^{\circ} \mathrm{C}$ with shaking at $200 \mathrm{rpm}$. Three colonies were selected as 3 biological replicates of each culture. An amount of $500 \mu \mathrm{L}$ overnight culture was centrifuged for $2 \mathrm{~min}$ at 10,000 rpm, washed twice and resuspended in $500 \mu \mathrm{L}$ SC medium. An aliquot of $10 \mu \mathrm{L}$ cell suspension was transferred to $988 \mu \mathrm{L}$ SC medium containing $2 \mu \mathrm{L}$ PEs (M. esculenta, W. bifurcata, T. divaricata leaf extracts) at different final concentrations of $0.1,1,5,10,50,100,200 \mu \mathrm{g} / \mathrm{mL}$ for each PE in a Bijou bottle [27]. As a control, $10 \mu \mathrm{L}$ cell suspension was transferred to $988 \mu \mathrm{L}$ SC medium containing $2 \mu \mathrm{L}$ DMSO in a Bijou bottle. The cells were incubated and aged at $30{ }^{\circ} \mathrm{C}$ with continuous shaking at $200 \mathrm{rpm}$ throughout the experiment. On day 0, 2, 4, and 6 of the stationary phase, an aliquot of $2 \mu \mathrm{L}$ of each aged culture was transferred into a 96-well flat-bottom microplate (IWAKI) containing $98 \mu \mathrm{L}$ YPD medium. The outgrowths of yeast cultures in the 96-well microplate were monitored by measuring the $A_{600}$ every 30 min until $24 \mathrm{~h}$ using Bio Microplate Reader HiTS (Cosmo Bio, Tokyo, Japan). Survival percentages and survival integrals of the cultures were calculated using the formulae stated earlier in the chronological life span assay. The analysis of significant differences in survival integrals was performed using one-way ANOVA with the Tukey test. Differences at $p<0.05$ were considered statistically significant.

\subsection{Stress Assay}

A single colony of yeast strain 1783 was selected from YPD agar and grown overnight in a Bijou bottle with $1 \mathrm{~mL}$ of YPD medium at $30^{\circ} \mathrm{C}$ with shaking at $200 \mathrm{rpm}$. At least 3 biological replicates of each PE-treated culture or control culture were prepared from different single colonies. An amount of $500 \mu \mathrm{L}$ overnight culture was centrifuged for $2 \mathrm{~min}$ at 10,000 rpm, washed twice and resuspended in $500 \mu \mathrm{L} \mathrm{SC}$ medium. An aliquot of $10 \mu \mathrm{L}$ cell suspension was transferred to $988 \mu \mathrm{L}$ SC medium containing $2 \mu \mathrm{L}$ PE (M. esculenta or W. bifurcata leaf extract) at a final concentration of 50 and $10 \mu \mathrm{g} / \mathrm{mL}$, respectively, in a Bijou bottle [27]. As a control, $10 \mu \mathrm{L}$ cell suspension was transferred to $988 \mu \mathrm{L}$ SC medium containing $2 \mu \mathrm{L}$ DMSO in a Bijou bottle. The cells were incubated and aged at $30{ }^{\circ} \mathrm{C}$ with continuous shaking at $200 \mathrm{rpm}$ throughout the experiment. On day $0,4,6$ and 10 of the stationary phase, an aliquot of $50 \mu \mathrm{L}$ of each aged culture was washed twice and resuspended in $50 \mu \mathrm{L}$ sterile distilled water. The aged culture suspensions were exposed to oxidative stress by being treated with $3 \mathrm{mM}$ hydrogen peroxide $\left(\mathrm{H}_{2} \mathrm{O}_{2}\right)$ for $1 \mathrm{~h}$ in the dark at $30{ }^{\circ} \mathrm{C}$ with shaking at $200 \mathrm{rpm}$. After $\mathrm{H}_{2} \mathrm{O}_{2}$ treatment, the cells were washed twice and resuspended in sterile distilled water. For thermal stress exposure, an amount of $50 \mu \mathrm{L}$ of each aged culture suspension was incubated in a water bath at $55^{\circ} \mathrm{C}$ for $20 \mathrm{~min}$ and were cooled at $30^{\circ} \mathrm{C}$ for $30 \mathrm{~min}$. A 10 -fold serial dilution $\left(10^{-2}, 10^{-3}, 10^{-4}, 10^{-5}\right)$ was conducted by diluting the non- and stress-treated cultures in distilled water. An amount of $10 \mu \mathrm{L}$ 10-fold serially diluted non- and stress-treated cultures were spotted on YPD agar in duplicate. The agar plates were incubated for 2 days at $30^{\circ} \mathrm{C}$. Colonies of the strains formed after 2 days were observed. For outgrowth measurement, an amount of $2 \mu \mathrm{L}$ of non- or stress-treated culture was transferred to a 96-well flat-bottom microplate (IWAKI) containing $98 \mu \mathrm{L}$ YPD medium on day $0,4,6$ and 10. The outgrowths of yeast cultures in the 96-well microplate were monitored by measuring the $A_{600}$ every 30 min until $24 \mathrm{~h}$ using Bio Microplate Reader HiTS (Cosmo Bio, Tokyo, Japan). Survival percentages of the cultures were calculated using the formulae stated earlier in the chronological life span assay.

\section{Results}

\subsection{Seven PEs Extend Chronological Life Span in Yeast}

To determine the effects of the PEs on yeast CLS, we used an adapted chronological life span assay for 96-well plate screening as previously reported [27]. Briefly, the yeast cells were treated with PEs at a final concentration of $100 \mu \mathrm{g} / \mathrm{mL}$ and were grown and 
aged in 96-well microplates containing SC media. To measure cell survival, an aliquot of ageing culture (on day 0, 2, 4 and 6) was transferred to a 96-well microplate containing YPD media for outgrowth. The absorbances of outgrowth cultures were recorded by a microplate reader for result analysis.

From a total of 222 PEs (Supplementary Table S2) screened in the primary screen, 45 PEs-treated cultures recorded higher relative absorbances than that of the control culture throughout the ageing period (Supplementary Figure S6) and were selected for secondary screening. Among the 45 PEs, seven PEs (Wodyetia bifurcata leaf extract, Thevetia peruviana leaf extract, Tabernaemontana divaricata leaf extract, Calotropis gigantea leaf extract, Alstonia angustiloba leaf extract, Manihot esculenta leaf extract, Ziziphus mauritiana leaf extract) were found to have an overall higher survival than the control culture during chronological ageing (Supplementary Figure S9 and Figure 1B). The CLS extensions by the seven PEs were further verified in a third confirmatory screen. The survivals of the seven PEs-treated cultures on individual days 2,4 and 6 were 1.11, 1.27 and 1.07 (W. bifurcata); 1.09, 1.18 and 1.01 (T. peruviana); 1.09, 1.21 and 0.99 (T. divaricata); 1.03, 1.11 and 1.12 (C. gigantea); 1.03, 1.07 and 1.07 (A. angustiloba); 1.01, 1.25 and 1.16 (M. esculenta), and 1.04, 1.18 and 1.24 (Z. mauritiana), -times of the survival of control culture, respectively (Figure 1B). The overall survival integrals (SI), which was the area under curve (AUC) of the survival curves, of the cultures were 1.19 (W. bifurcata); 1.10 (T. peruviana); 1.26 (T. divaricata); 1.11 (C. gigantea); 1.06 (A. angustiloba); 1.16 (M. esculenta), and 1.08 (Z. mauritiana), -times of the survival integral of control culture, respectively (Figure 1B). The top three CLS-extending PEs (T. divaricata, W. bifurcata, and M. esculenta) were selected for further characterisation in the dose-dependent and stress assays.

\subsection{M. esculenta and W. bifurcata Extracts Provide Dose-Dependent Effects in Extending Yeast CLS}

To evaluate the dose-dependent effects of PEs on yeast CLS, the cells were treated with M. esculenta, W. bifurcata or T. divaricata leaf extract at different final concentrations of $0.1,1,5,10,50,100,200 \mu \mathrm{g} / \mathrm{mL}$ and aged in Bijou bottles containing SC media. On day 0, 2, 4 and 6, an aliquot of the PE-treated ageing culture was transferred to a 96-well microplate containing YPD media for outgrowth measured by a microplate reader.

The survival of yeast cells treated with $M$. esculenta leaf extract at $\leq 50 \mu \mathrm{g} / \mathrm{mL}$ was beyond $50 \%$, higher than that of the control throughout the ageing period while the survival of yeast cells treated with $M$. esculenta leaf extract at $\geq 100 \mu \mathrm{g} / \mathrm{mL}$ was less than $50 \%$, lower than the control on day 4 and 6 (Figure 2). To compare the extract's CLSextending activity across different concentrations tested, the survival integral, which was the AUC of the survival curve, was constructed. The survival integrals of yeast cells treated with $M$. esculenta leaf extract at $\leq 50 \mu \mathrm{g} / \mathrm{mL}$ were higher than that of the control culture $\left({ }^{* *} p<0.01 ;{ }^{* * *} p<0.001\right)$ while the survival integrals of yeast cells treated with M. esculenta extract at $\geq 100 \mu \mathrm{g} / \mathrm{mL}$ were not significantly different compared to that of the control cultures. The yeast cells treated with M. esculenta extract at $50 \mu \mathrm{g} / \mathrm{mL}$ had the highest survival integral, which was 1.42-times of that of the control culture (Figure 2).

The survivals of yeast cultures treated with $W$. bifurcata leaf extract ranged from $1 \mu \mathrm{g} / \mathrm{mL}$ to $100 \mu \mathrm{g} / \mathrm{mL}$ were higher than that of the DMSO control throughout the ageing period while the survivals of yeast cultures treated with $W$. bifurcata leaf extract at $0.1 \mu \mathrm{g} / \mathrm{mL}$ and $200 \mu \mathrm{g} / \mathrm{mL}$ were lower than that of the control on day 2 and 4 . The comparison of CLS-extending activity between groups of cultures treated with W. bifurcata leaf extract at different concentrations and the control was at a weak significant difference, $p=0.07$. Nevertheless, there is a trend of increasing survival integrals of the yeast cultures treated with $W$. bifurcata leaf extract plateauing in the range of $5 \mu \mathrm{g} / \mathrm{mL}$ to $10 \mu \mathrm{g} / \mathrm{mL}$, followed by a decrease with increasing concentrations of the extract above $50 \mu \mathrm{g} / \mathrm{mL}$ when compared to the DMSO control. The $10 \mu \mathrm{g} / \mathrm{mL}$ of W. bifurcata extract was found to have the highest survival integral of 1.36-times the control (Figure 2). 
A

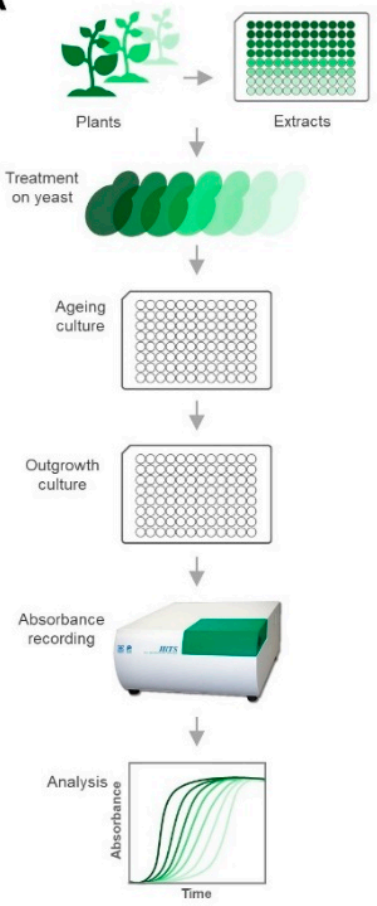

B
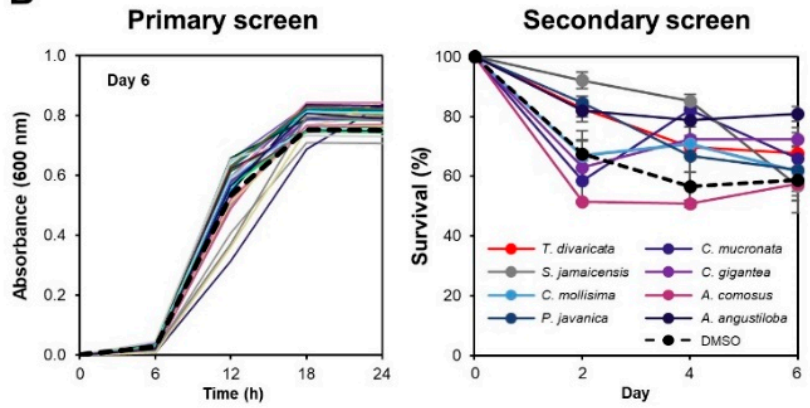

Confirmatory screen

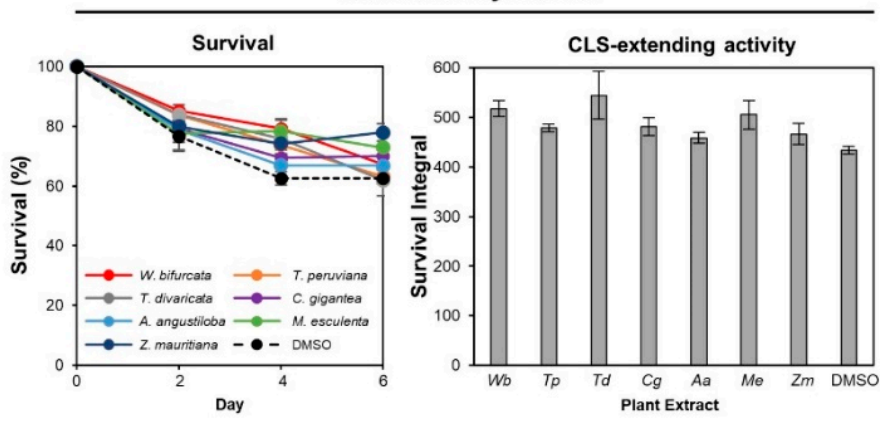

Figure 1. Screening for plant extracts (PEs) that extend yeast chronological life span (CLS). (A) Overview of the screening for PEs that extend S. cerevisiae CLS. Yeast cells of strain 1783 treated with various PEs or DMSO control were grown and aged in a 96-well microplate containing SC media. An aliquot of each aged culture was transferred to a new 96-well microplate containing fresh YPD medium to screen the PEs. The outgrowths of yeast cells were monitored by measuring their absorbances $\left(\mathrm{A}_{600}\right)$ using Bio Microplate Reader HiTS on alternate days. Growth curves of absorbance against time of outgrowth were plotted. (B) Representative results of the primary, secondary and confirmatory screens. In the primary screen, the absorbance values of 222 PEs-treated cultures at the 12th $\mathrm{h}$ from the outgrowth curves (Supplementary Figures S1-S5, Tables S3-S6) were used to calculate the relative absorbance values. The relative absorbance values were calculated by dividing the PE-treated cultures' average outgrowth absorbance readings at the 12th $\mathrm{h}$ to that of the DMSO control culture. Three biological replicates of each culture were prepared. In the secondary screen, the survival percentages of 45 PEs-treated cultures selected from the primary screen (Supplementary Figure S9) were derived from the outgrowth curves (Supplementary Figures S7 and S8). Error bars represent the standard errors of means (SEM) of three biological replicates. In the confirmatory screen, the survival percentages and survival integrals of seven PEs-treated cultures selected from the secondary screen were derived from the outgrowth curves (Supplementary Figure S10). Error bars represent the SEM of three biological replicates.

Intriguingly, the survivals of yeast cultures treated with the $T$. divaricata extract were not significantly different at all the tested concentrations compared to the DMSO control. This may be due to the different culture conditions compared to the screening stage. Therefore, the T. divaricata extract was excluded for further characterisation. Overall, the M. esculenta and $W$. bifurcata leaf extracts at $50 \mu \mathrm{g} / \mathrm{mL}$ and $10 \mu \mathrm{g} / \mathrm{mL}$ respectively were found to result in the highest survival integral and were selected for further characterisation. 

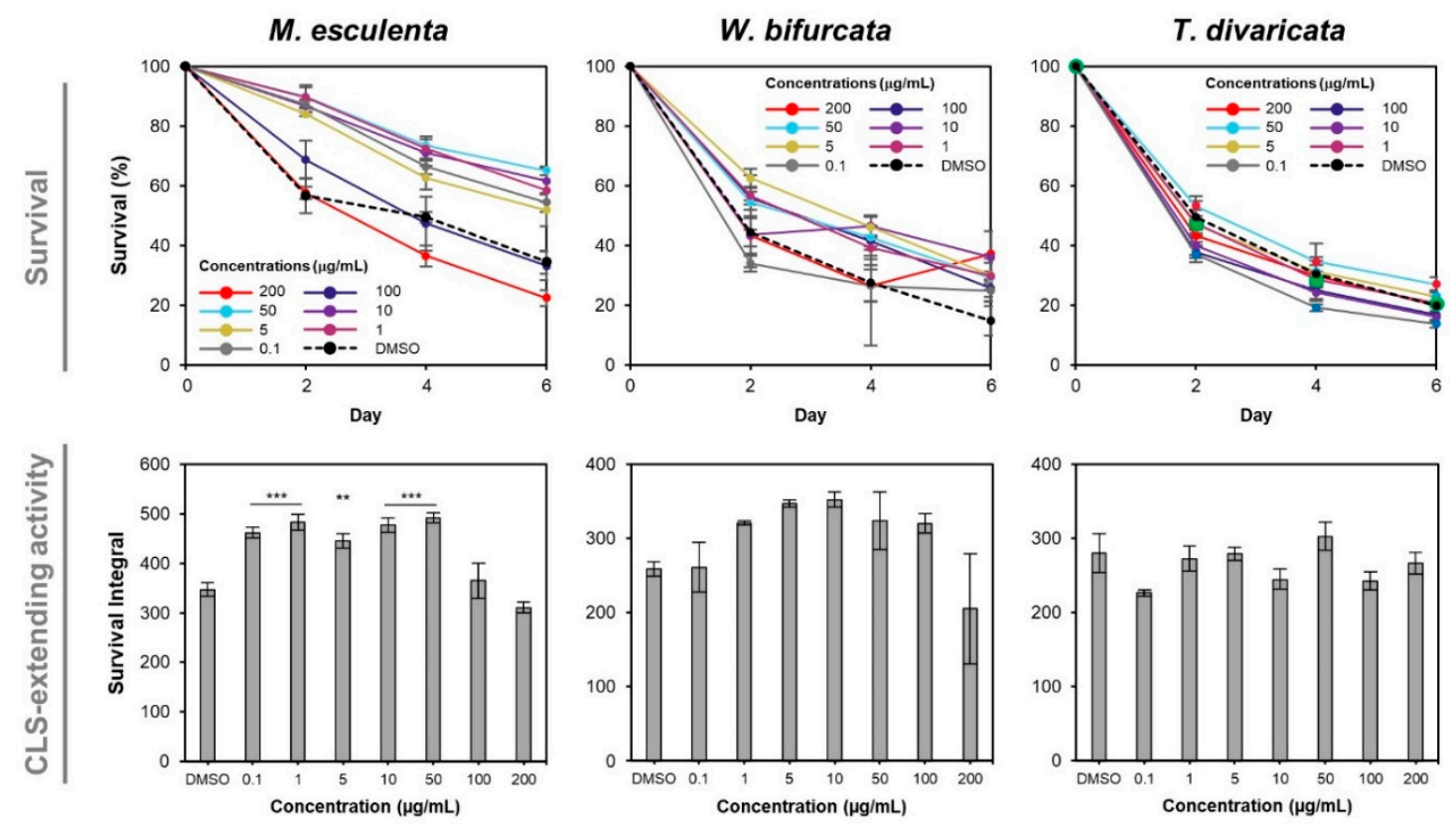

Figure 2. Dose-dependent effects of M. esculenta, W. bifurcata and T. divaricata extracts on yeast CLS. Yeast cells of strain 1783 treated with different concentrations of M. esculenta, W. bifurcata or T. divaricata leaf extract, or DMSO control were chronologically aged in Bijou bottles containing SC media until day 6. An aliquot of each aged culture was transferred to a 96-well microplate containing YPD medium for outgrowth on day 0, 2, 4 and 6. The survival percentages and survival integrals of the cultures were derived from the outgrowth curves (Supplementary Figure S11). Tukey test was performed (IBM SPSS Statistics software). The survival integrals of yeast cells treated with M. esculenta only at 0.1, 1, 5, 10,50 $\mu \mathrm{g} / \mathrm{mL}$ were significantly different from that of the DMSO control cells $\left(^{* *} p<0.01\right.$; $\left.{ }^{* * *} p<0.001\right)$. Error bars represent the SEM of three biological replicates.

\subsection{M. esculenta and W. bifurcata Extracts Contribute to Oxidative Stress Tolerance during Chronological Ageing}

To evaluate the stress response of yeast cultures treated with M. esculenta or W. bifurcata leaf extracts, we used the optimal concentration of $50 \mu \mathrm{g} / \mathrm{mL}$ and $10 \mu \mathrm{g} / \mathrm{mL}$, respectively, identified from the dose-dependent assay. The PE-treated cultures were aged in Bijou bottles containing SC media as before, then the aged cultures were subjected to oxidative stress by the addition of $3 \mathrm{mM}$ hydrogen peroxide $\left(\mathrm{H}_{2} \mathrm{O}_{2}\right)$ or to thermal stress by incubation at $55^{\circ} \mathrm{C}$. Spot assays were performed to observe the growth phenotype and an outgrowth assay was also used to quantify the cell survival upon post-stress treatment.

In the spot assay, which was a semi-quantitative assay (Figure 3), we observed that the yeast cultures treated with $M$. esculenta leaf extract had a lower survival compared to the non-treated respective yeast cultures by forming less colonies at $10^{-4}$ and $10^{-5}$ dilutions starting from day 0 and forming colonies only up to $10^{-3}$ dilution on day 10 after oxidative or thermal stress exposure.

In the case of the yeast cultures treated with W. bifurcata leaf extract, they had lower survival compared to the non-treated yeast cultures by forming fewer colonies at $10^{-4}$ and $10^{-5}$ dilutions starting from day 0 and forming colonies only up to $10^{-4}$ dilution on day 6 after oxidative stress and forming colonies only up to $10^{-3}$ dilution on day 4 , up to $10^{-4}$ dilution on day 6 , and no colonies were formed on day 10 after thermal stress exposure. For the DMSO control cultures, they had lower survival compared to the nonstress treated respective yeast cultures by forming less colonies at $10^{-4}$ and $10^{-5}$ dilutions on day 0,6 and 10 and forming colonies only up to $10^{-4}$ dilution on day 0 after oxidative stress exposure and forming fewer colonies at $10^{-3}$ and $10^{-4}$ dilution starting from day 4 after thermal stress exposure. Overall, the growth phenotypes of the PEs-treated or DMSO control cultures were affected negatively after stress exposure during chronological ageing. 


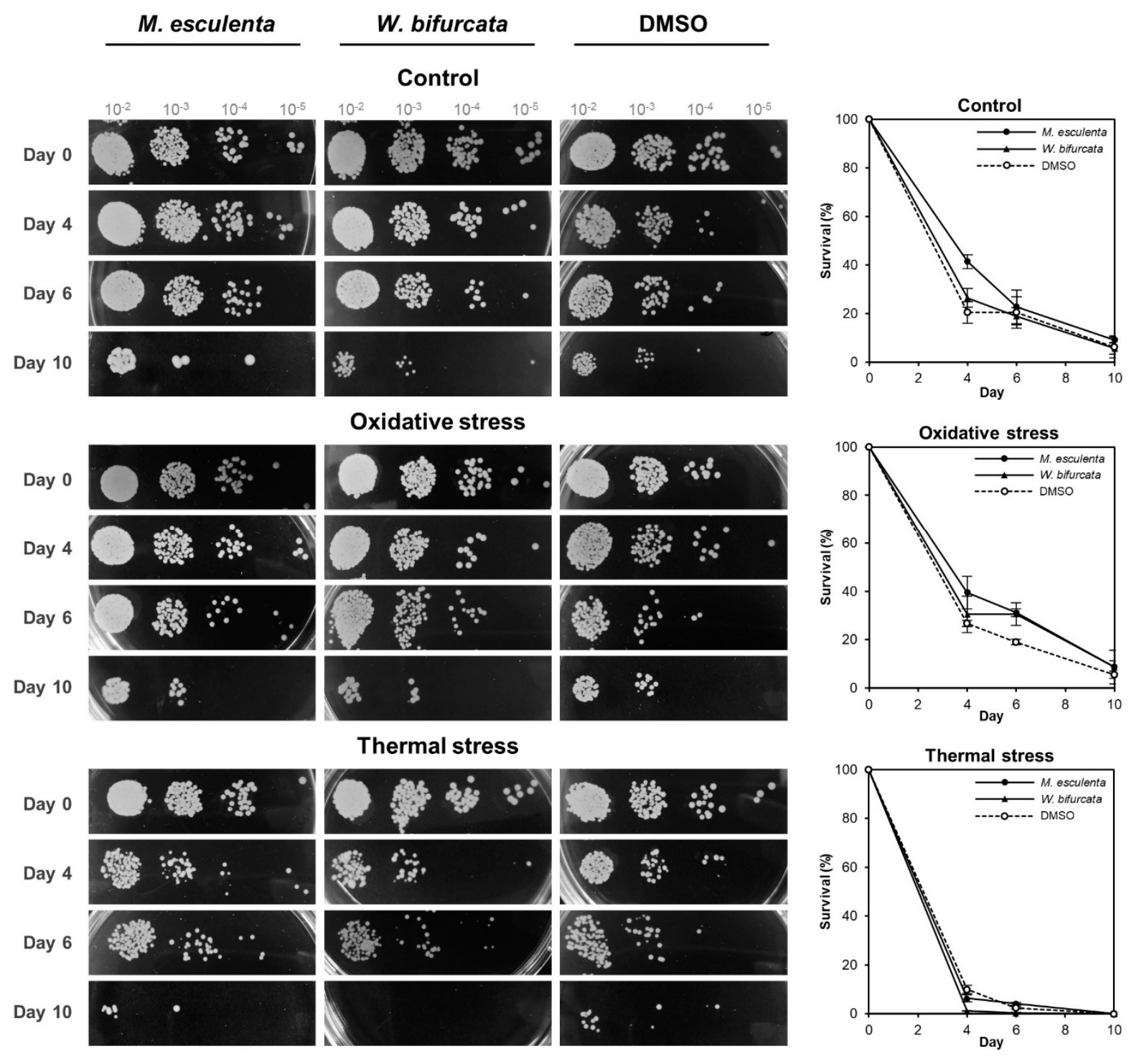

Figure 3. Oxidative and thermal stress responses of PEs-treated yeast and control yeast during chronological ageing. Yeast cultures of strain 1783 treated with M. esculenta or W. bifurcata leaf extract at optimal final concentrations of $50 \mu \mathrm{g} / \mathrm{mL}$ and $10 \mu \mathrm{g} / \mathrm{mL}$, respectively, or DMSO control were chronologically aged in Bijou bottles until day 10. At each age point, a small volume of yeast cells was transferred out of the ageing culture and was exposed to oxidative stress by being treated with $3 \mathrm{mM} \mathrm{H}_{2} \mathrm{O}_{2}$ for $1 \mathrm{~h}$ at $30^{\circ} \mathrm{C}$ or thermal stress at $55^{\circ} \mathrm{C}$ for $20 \mathrm{~min}$ before being spotted in 10 -fold serial dilutions $\left(10^{-2}, 10^{-3}\right.$, $10^{-4}, 10^{-5}$ ) on YPD agar. Quantitative outgrowth CLS analysis was carried out simultaneously to determine the survival of cultures aged with M. esculenta or W. bifurcata leaf extracts after oxidative and thermal stress exposure. An aliquot of each non- or stress-treated culture was transferred to a 96-well microplate containing YPD medium for outgrowth on day 0, 4, 6 and 10. The survival percentages of the cultures were derived from the outgrowth curves (Supplementary Figure S12). Error bars represent the SEM of at least three (three to six) biological replicates.

To quantitate the cell survival upon stress exposure, we also performed an outgrowth kinetics assay (Figure 3, right panels). We previously demonstrated that cell number was directly related to absorbance $\left(\mathrm{OD}_{600}\right)$ [27]. In the non-stress treated, M. esculenta leaf extract extended CLS in budding yeast throughout the ageing period agreeing with dose-dependent result, however the W. bifurcata extract did not extend CLS beyond day 6 . Both M. esculenta and W. bifurcata leaf extracts contributed to oxidative stress tolerance with a higher survival percentage than the DMSO control culture. However, they did not appear to contribute to thermal stress tolerance. These results indicate that $M$. esculenta 
and $W$. bifurcata leaf extracts may potentially be involved in the cellular oxidative stress response pathway to extend CLS in budding yeast.

\section{Discussion}

Various plants are known to contain ageing-delaying phytochemicals. Here, we report the identification of two PEs that have not been reported before that can extend S. cerevisiae life span. Specifically, we first developed a semi-high-throughput 96-well plate-based CLS assay, screened 222 PEs from 70 plant families, and identified two PEs that can extend yeast CLS possibly by conferring protection against oxidative stress.

S. cerevisiae has been widely used as a model organism for the discovery of PEs and small molecules that can extend CLS by using various cell viability protocols. For example, Melannurca Campana apple extracts were identified to extend yeast CLS through the microcolony scoring method [28]. The yeast CLS extension by pigmented Hom Dang rice bran extracts was determined using a methylene blue-stained cell counting method [29]. While those methods are useful in their respective studies, the laborious and time-consuming methods of microscopic colony count do not allow for parallel screening of multiple PEs simultaneously. The microplate-based yeast ageing and screening assay using the outgrowth method is a practical option that allows the identification of candidate PEs for further verification.

Our CLS screen first identified seven candidate PEs (W. bifurcata leaf extract, T. peruviana leaf extract, T. divaricata leaf extract, C. gigantea leaf extract, A. angustiloba leaf extract, M. esculenta leaf extract, and Z. mauritiana leaf extract) that could extend yeast CLS. These seven PEs with the presence of phytochemicals such as flavonoids, tannins, caratenoids, terpenoids, saponins and alkaloids, have been reported to exert various beneficial biological activities such as antibacterial activity, antiproliferative effect against cancer cell lines, antioxidant activity and so on in vitro and in vivo (Table 1).

Table 1. Phytochemical profiles and previously reported biological activities of seven PEs were identified from the CLS assay.

\begin{tabular}{|c|c|c|c|}
\hline PE (Organ) & Phytochemical & Biological Activity & Reference \\
\hline Wodyetia bifurcate (leaf) & $\begin{array}{l}\text { Methanol and butanol extracts contain } \\
\text { triterpene, flavonoids, benzenoid and } \\
\text { polyphenol }\end{array}$ & $\begin{array}{l}\text { Weak cytotoxicity against human liver Hep- } \mathrm{G}_{2} \\
\text { cancer cell line }\end{array}$ & [30] \\
\hline Thevetia peruviana (leaf) & $\begin{array}{l}\text { Contains alkaloids, cardiac glycosides, } \\
\text { flavonoids, polyphenols, saponins and } \\
\text { tannins }\end{array}$ & $\begin{array}{l}\text { Antibacterial activity against foodborne } \\
\text { microorganisms; treatment for a diarrhoea-induced } \\
\text { rat model }\end{array}$ & {$[31,32]$} \\
\hline Tabernaemontana divaricate (leaf) & Diverse in alkaloids and non-alkaloids & $\begin{array}{c}\text { Mild insecticides against crop pests; folk medicine; } \\
\text { mimics the effect of acetylcholinesterase inhibitors } \\
\text { towards Alzheimer's disease }\end{array}$ & [33-35] \\
\hline Calotropis gigantean (leaf) & $\begin{array}{l}\text { Hydro-alcoholic extracts contain } \\
\text { flavonoid, tannins, alkaloids, and } \\
\text { steroids }\end{array}$ & $\begin{array}{l}\text { Traditional medicine; antifungal against Candida sp.; } \\
\text { antibacterial activity towards Escherichia coli., } \\
\text { Pseudomonas aeruginosa and Bacillus cereus; } \\
\text { antiproliferative effect against human cancer cell lines } \\
\text { (MCF7, MDA-MB-231, and HeLa); antioxidant activity }\end{array}$ & [36-39] \\
\hline Alstonia angustiloba (leaf) & Ethanol extracts contain alkaloids & $\begin{array}{l}\text { Weak antiproliferative against human cancer cell } \\
\text { lines (MCF7, MDA-MB-231, and HeLa) }\end{array}$ & {$[39,40]$} \\
\hline Manihot esculenta (leaf) & $\begin{array}{l}\text { Ethanol extracts contain terpenoids, } \\
\text { flavonoids, carotenoids and tannins }\end{array}$ & $\begin{array}{l}\text { In vivo anti-inflammatory on rat model with paw } \\
\text { oedema; contains antipyretic activity, which mimics the } \\
\text { effect of paracetamol; anthelmintic activity against } \\
\text { gastrointestinal parasite Haemonchus contortus }\end{array}$ & {$[41,42]$} \\
\hline Ziziphus mauritiana (stem) & $\begin{array}{l}\text { Various solvent extraction contains } \\
\text { alkaloids, flavonoids, tannins, } \\
\text { carotenoids, saponins, steroids, } \\
\text { triterpenoids and anthraquinones }\end{array}$ & $\begin{array}{l}\text { Traditional medicine; in vivo anti-ulcers against aspirin } \\
\text { and ethanol ulcers in mice model; detoxification of } \\
\text { silica-induced toxicity in the liver of Albino rats; } \\
\text { antibacterial against pathogens such as Salmonella typhi, } \\
\text { Bacillus subtilis and Staphylococcus aureus }\end{array}$ & {$[12,43,44]$} \\
\hline
\end{tabular}

Among the seven candidate PEs, the three leaf extracts of M. esculenta, W. bifurcata, and T. divaricata extended yeast CLS the most. Next, we further evaluated the dose-dependent effects of the top three CLS-extending PEs during chronological ageing. Among these three PEs, M. esculenta and $W$. bifurcata leaf extracts were dose-dependent in their extension of yeast CLS. However, T. divaricata leaf extracts did not show the same effect. This could be 
due to the different ageing culture incubation methods (non-shaking microplate-based or shaking bottle-based methods) in both assays.

Ageing can be caused by environmental stress [45]. Oxidative stress, which is a major contributor to ageing, occurs when ROS accumulate in cells. ROS such as hydrogen peroxide $\left(\mathrm{H}_{2} \mathrm{O}_{2}\right)$, superoxide anion $\left(\mathrm{O}_{2}{ }^{-}\right)$and hydroxyl free radical $(\mathrm{OH} \cdot)$ can cause oxidative damage of biomacromolecules $[46,47]$. We found that M. esculenta and W. bifurcata leaf extracts conferred oxidative stress tolerance to the ageing cells. M. esculenta extracts had been reported to possess antioxidant activity against lipid peroxidation and mild capability in capturing free radicals [48]. Additionally, M. esculenta extract contains carotenoid and flavonoid [42], which are well-known antioxidants in protecting cells from harmful ROS [49-52]. W. bifurcata extract also contains flavonoid [30], which acts as an antioxidant as well [51,52]. Flavonoid compounds consist of hydroxyl groups, which the hydrogens and electrons can be donated to ROS superoxide anion then further reduced into unharmed water and oxygen molecules [53]. Therefore, both M. esculenta and W. bifurcata extracts likely extended yeast CLS through the presence of antioxidant bioactive compounds that protected yeast cells from oxidative stress during chronological ageing. This agrees with studies that showed that PEs from apple, citrus peel and pigmented rice bran delay ageing by contributing resistance against oxidative stress in S. cerevisiae $[28,29,54]$.

Thermal stress can cause cell death as the cells experience downregulated thermal stress response and thus accumulate damaged proteins and misfolded protein during ageing [45]. It was suggested that PEs could possibly extend yeast CLS through various longevity-relating cellular activities such as elevating oxidative and thermal stress tolerance [19]. However, both M. esculenta and W. bifurcata extracts did not confer thermal tolerance under the conditions that we tested $\left(55^{\circ} \mathrm{C}\right.$ for $\left.20 \mathrm{~min}\right)$. Furthermore, the survivals of the thermal-stressed cells were reduced by more than $50 \%$ compared to the non-stressed control.

In summary, we identified two PEs, M. esculenta and W. bifurcata, that extended yeast CLS possibly through the conferment of oxidative stress tolerance from carotenoid and flavonoid compounds that are present in the extracts. Further studies are needed to determine the effects of these PEs on various aspects of cellular processes such as the regulation of longevity-defined pathways (TOR and PKA pathways), mitochondrial respiration and lipid peroxidation in order to understand and elucidate the specific effects these PEs may have on longevity.

Supplementary Materials: The following are available online at https:/ /www.mdpi.com/article/ 10.3390/cells10102718/s1, Table S1: final concentrations of amino acids in SC medium, Table S2: list of 222 plant extracts used for primary CLS assay screen, Table S3: absorbance values $\left(\mathrm{A}_{600}\right)$ of yeast cultures treated with PEs (P1 A1 until P1 H11) at the 12th h from day 0 to day 6 in primary screen, Table S4: absorbance values ( $\mathrm{A}_{600}$ ) of yeast cultures treated with PEs (P2 A1 until P2 D11) at 12th $\mathrm{h}$ from day 0 to day 6 in primary screen, Table S5: absorbance values $\left(\mathrm{A}_{600}\right)$ of yeast cultures treated with PEs (P2 E1 until P2 H12) at the 12th $\mathrm{h}$ from day 0 to day 6 in primary screen, Table S6: absorbance values $\left(\mathrm{A}_{600}\right)$ of yeast cultures treated with PEs (P3 A1 until P3 C12) at the 12th $\mathrm{h}$ from day 0 to day 6 in primary screen, Figure S1: CLS outgrowth curves of yeast treated with PEs (P1 A1 until P1 D12) in primary screen, Figure S2: CLS outgrowth curves of yeast treated with PEs (P1 E1 till P1 H11) in primary screen, Figure S3: CLS outgrowth curves of yeast treated with PEs (P2 A1 until P2 D11) in primary screen, Figure S4: CLS outgrowth curves of yeast treated with PEs (P2 E1 until P2 H12) in primary screen, Figure S5: CLS outgrowth curves of yeast treated with PEs (P3 A1 until P3 C12) in primary screen, Figure S6: relative absorbance values $\left(\mathrm{A}_{600}\right)$ of 45 PEs-treated cultures, which are higher than that of the DMSO control cultures at the 12th $\mathrm{h}$ from day 0 to day 6 in primary screen, Figure S7: CLS outgrowth curves of yeast treated with PEs in secondary screen, Figure S8: CLS outgrowth curves of yeast treated with PEs in secondary screen, Figure S9: survival of yeast treated with 45 PEs in secondary screen, Figure S10: CLS outgrowth curves of yeast treated with seven PEs in confirmatory screen, Figure S11: CLS outgrowth curves of yeast treated with three PEs in dose-dependent assay, Figure S12: CLS outgrowth curves of yeast treated with two PEs in stress assays. 
Author Contributions: Conceptualization, M.M.Y.K., H.A.W., N.W. and E.B.B.O.; methodology, M.M.Y.K., H.A.W., N.W. and E.B.B.O.; validation, M.M.Y.K., J.W.L., H.A.W. and E.B.B.O.; formal analysis, M.M.Y.K. and E.B.B.O.; investigation, M.M.Y.K. and E.B.B.O.; resources, M.R.S., H.A.W., N.W. and E.B.B.O.; data curation, M.M.Y.K. and E.B.B.O.; writing-original draft preparation, M.M.Y.K. and J.W.L.; writing-review and editing, M.M.Y.K., J.W.L. and E.B.B.O.; visualization, M.M.Y.K., J.W.L. and E.B.B.O.; supervision, H.A.W., N.W. and E.B.B.O.; project administration, M.R.S. and E.B.B.O.; funding acquisition, M.R.S. and E.B.B.O. All authors have read and agreed to the published version of the manuscript.

Funding: This research was funded by Universiti Sains Malaysia RU Top-Down grant (1001/CIPPM/ 870038) for the USM-RIKEN International Centre for Ageing Science (URICAS) programme.

Institutional Review Board Statement: Not applicable.

Informed Consent Statement: Not applicable.

Data Availability Statement: The data presented in this study are available on request from the corresponding author.

Acknowledgments: The authors acknowledge the support provided by Universiti Sains Malaysia and RIKEN for the URICAS programme.

Conflicts of Interest: The authors declare no conflict of interest.

\section{References}

1. Lakatta, E.G.; Mitchell, J.H.; Pomerance, A.; Rowe, G.G. Human aging: Changes in structure and function. J. Am. Coll. Cardiol. 1987, 10, 42A-47A. [CrossRef]

2. Chodzko-Zajko, W.J. The physiology of aging: Structural changes and functional consequences. Implications for research and clinical practice in the exercise and activity sciences. Quest 1996, 48, 311-329. [CrossRef]

3. Viña, J.; Borrás, C.; Miquel, J. Theories of ageing. IUBMB Life 2007, 59, 249-254. [CrossRef] [PubMed]

4. Rodríguez-Rodero, S.; Fernández-Morera, J.L.; Menéndez-Torre, E.; Calvanese, V.; Fernández, A.F.; Fraga, M.F. Aging genetics and aging. Aging Dis. 2011, 2, 186-195.

5. Lee, J.W.; Ong, E.B.B. Genomic instability and cellular senescence: Lessons from the budding yeast. Front. Cell Dev. Biol. 2021, 8, 619126. [CrossRef]

6. Murakami, C.J.; Burtner, C.R.; Kennedy, B.K.; Kaeberlein, M. A method for high-throughput quantitative analysis of yeast chronological life span. J. Gerontol. A Biol. Sci. Med. Sc. 2008, 63A, 113-121. [CrossRef] [PubMed]

7. Sampaio-Marques, B.; Burhans, W.C.; Ludovico, P. Longevity pathways and maintenance of the proteome: The role of autophagy and mitophagy during yeast ageing. Microb. Cell 2014, 1, 118-127. [CrossRef] [PubMed]

8. Fabrizio, P.; Longo, V.D. The chronological life span of Saccharomyces cerevisiae. Aging Cell 2003, 2, 73-81. [CrossRef] [PubMed]

9. Longo, V.D.; Shadel, G.S.; Kaeberlein, M.; Kennedy, B. Replicative and chronological aging in Saccharomyces cerevisiae. Cell Metab. 2012, 16, 18-31. [CrossRef]

10. Polymenis, M.; Kennedy, B.K. Chronological and replicative lifespan in yeast: Do they meet in the middle? Cell Cycle 2012, 11, 3531. [CrossRef] [PubMed]

11. Wilson, M.A.; Shukitt-Hale, B.; Kalt, W.; Ingram, D.K.; Joseph, J.A.; Wolkow, C.A. Blueberry polyphenols increase lifespan and thermotolerance in Caenorhabditis elegans. Aging Cell 2006, 5, 59-68. [CrossRef]

12. Talmale, S.A.; Bhujade, A.M.; Patil, M.B. Phytochemical analysis of stem bark and root bark of Zizyphus mauritiana. Int. J. Innov. Sci. Eng. Technol. 2014, 1, 526-535.

13. Saxena, M.; Saxena, J.; Nema, R.; Singh, D.; Gupta, A. Phytochemistry of medicinal plants. J. Pharmacogn. Phytochem. 2013, $1,168-182$.

14. Corrêa, R.C.G.; Peralta, R.M.; Haminiuk, C.W.I.; Maciel, G.M.; Bracht, A.; Ferreira, I.C.F.R. New phytochemicals as potential human anti-aging compounds: Reality, promise, and challenges. Crit. Rev. Food Sci. Nutr. 2018, 58, 942-957. [CrossRef]

15. Zhang, J.; Xiao, Y.; Guan, Y.; Rui, X.; Zhang, Y.; Dong, M.; Ma, W. An aqueous polyphenol extract from Rosa rugosa tea has antiaging effects on Caenorhabditis elegans. J. Food Biochem 2019, 43, e12796. [CrossRef]

16. Leite, N.R.; Araújo, L.C.A.D.; Rocha, P.D.S.D.; Agarrayua, D.A.; Ávila, D.S.; Carollo, C.A.; Silva, D.B.; Estevinho, L.M.; de Picoli Souza, K.; dos Santos, E.L. Baru pulp (Dipteryx alata Vogel): Fruit from the Brazilian savanna protects against oxidative stress and increases the life expectancy of Caenorhabditis elegans via SOD-3 and DAF-16. Biomolecules 2020, 10, 1106. [CrossRef]

17. Pérez-Gálvez, A.; Viera, I.; Roca, M. Carotenoids and chlorophylls as antioxidants. Antioxidants 2020, 9, 505. [CrossRef]

18. Dakik, P.; Rodriguez, M.E.L.; Junio, J.A.B.; Mitrofanova, D.; Medkour, Y.; Tafakori, T.; Taifour, T.; Lutchman, V.; Samson, E.; Arlia-Ciommo, A.; et al. Discovery of fifteen new geroprotective plant extracts and identification of cellular processes they affect to prolong the chronological lifespan of budding yeast. Oncotarget 2020, 11, 2192-2213. [CrossRef] [PubMed] 
19. Lutchman, V.; Medkour, Y.; Samson, E.; Arlia-Ciommo, A.; Dakik, P.; Cortes, B.; Feldman, R.; Mohtashami, S.; McAuley, M.; Chancharoen, M.; et al. Discovery of plant extracts that greatly delay yeast chronological aging and have different effects on longevity-defining cellular processes. Oncotarget 2016, 7, 16542-16566. [CrossRef]

20. Powers III, R.W.; Kaeberlein, M.; Caldwell, S.D.; Kennedy, B.K.; Fields, S. Extension of chronological life span in yeast by decreased TOR pathway signaling. Genes Dev. 2006, 20, 174-184. [CrossRef] [PubMed]

21. Wei, M.; Fabrizio, P.; Hu, J.; Ge, H.; Cheng, C.; Li, L.; Longo, V.D. Life span extension by calorie restriction depends on Rim15 and transcription factors downstream of Ras/PKA, Tor, and Sch9. PLoS Genet. 2008, 4, 0139-0149. [CrossRef]

22. Huang, X.; Liu, J.; Dickson, R.C. Down-regulating sphingolipid synthesis increases yeast lifespan. PLoS Genet. 2012, 8. [CrossRef] [PubMed]

23. Wierman, M.B.; Maqani, N.; Strickler, E.; Li, M.; Smith, J.S. Caloric restriction extends yeast chronological life span by optimizing the Snf1 (AMPK) signaling pathway. Mol. Cell Biol. 2017, 37, e00562-16. [CrossRef]

24. Lin, Y.; Kotakeyama, Y.; Li, J.; Pan, Y.; Matsuura, A.; Ohya, Y.; Yoshida, M.; Xiang, L.; Qi, J. Cucurbitacin B exerts antiaging effects in yeast by regulating autophagy and oxidative stress. Oxid. Med. Cell. Longev. 2019, 2019, 4517091. [CrossRef]

25. Martinez-Lopez, N.; Athonvarangkul, D.; Singh, R. Longevity Genes: A Blueprint for Aging; Atzmon, G., Ed.; Springer: New York, NY, USA, 2015; pp. 73-87.

26. Lutchman, V.; Dakik, P.; McAuley, M.; Cortes, B.; Ferraye, G.; Gontmacher, L.; Graziano, D.; Moukhariq, F.-Z.; Simard, É; Titorenko, V.I. Six plant extracts delay yeast chronological aging through different signaling pathways. Oncotarget 2016, 7, 50845-50863. [CrossRef] [PubMed]

27. Kwong, M.M.Y.; Lee, J.W.; Samian, M.R.; Watanabe, N.; Osada, H.; Ong, E.B.B. Comparison of microplate- and bottle-based methods to age yeast for chronological life span assays. J. Microbiol. Methods 2019, 167, 105743. [CrossRef]

28. Stirpe, M.; Palermo, V.; Bianchi, M.M.; Silvestri, R.; Falcone, C.; Tenore, G.; Novellino, E.; Mazzoni, C. Annurca apple (M. pumila Miller cv Annurca) extracts act against stress and ageing in S. cerevisiae yeast cells. BMC Complement. Altern Med. 2017, 17, 200. [CrossRef]

29. Sunthonkun, P.; Palajai, R.; Somboon, P.; Suan, C.L.; Ungsurangsri, M.; Soontorngun, N. Life-span extension by pigmented rice bran in the model yeast Saccharomyces cerevisiae. Sci. Rep. 2019, 9, 18061. [CrossRef]

30. Sengab, A.E.B.; El naggar, D.M.Y.; Elgindi, M.R.; Elsaid, M.B. Biological studies of isolated triterpenoids and phenolic compounds identified from Wodyetia bifurcata family Arecaceae. J. Pharmacogn. Phytochem. 2015, 3, 67-73.

31. Hassan, M.M.; Saha, A.K.; Khan, S.A.; Islam, A.; Mahabub-Uz-Zaman, M.; Ahmed, S.S.U. Studies on the antidiarrhoeal, antimicrobial and cytotoxic activities of ethanol-extracted leaves of yellow oleander (Thevetia Peruviana). Open Vet. J. 2011, 1, 28-31. [PubMed]

32. Gezahegn, Z.; Akhtar, M.S.; Woyessa, D.; Tariku, Y. Antibacterial potential of Thevetia Peruviana leaf extracts against food associated bacterial pathogens. J. Coast. Life Med. 2015, 3, 150-157. [CrossRef]

33. Van Beek, T.A.; Verpoorte, R.; Svendsen, A.B.; Leeuwenberg, A.J.M.; Bisset, N.G. Tabernaemontana L. (Apocynaceae): A review of its taxonomy, phytochemistry, ethnobotany and pharmacology. J. Ethnopharmacol. 1984, 10, 1-156. [CrossRef]

34. Pratchayasakul, W.; Pongchaidecha, A.; Chattipakorn, N.; Chattipakorn, S.C. Reversible acetylcholinesterase inhibitory effect of Tabernaemontana divaricata extract on synaptic transmission in rat CA1 hippocampus. Indian J. Med. Res. 2010, 131, 411-417. [PubMed]

35. Islam, M.S.; Islam, N.; Ahsan, M.K.; Mahdi, S.H.A. Bio-efficacy of Tabernaemontana Divaricata (L.) leaf and stem bark extract against Callosobruchus chinensis L. Sch. Acad. J. Biosci. 2018, 6, 247-251. [CrossRef]

36. Kumar, G.; Karthik, L.; Rao, K.V.B. Antibacterial activity of aqueous extract of Calotropis gigantea leaves-An in vitro study. Int. J. Pharm. Sci. Rev. Res. 2010, 4, 141-144.

37. Kumar, G.; Karthik, L.; Rao, K.V.B. In vitro anti-Candida activity of Calotropis gigantea. J. Pharm. Res. $2010,3,539-542$.

38. Singh, N.; Jain, N.K.; Kannojia, P.; Garud, N.; Pathak, A.K.; Mehta, S.C. In vitro antioxidant activity of Calotropis gigantea hydroalcohlic leaves extract. Der Pharm. Lett. 2010, 2, 95-100.

39. Wong, S.; Lim, Y.; Abdullah, N.; Nordin, F. Antiproliferative and phytochemical analyses of leaf extracts of ten Apocynaceae species. Pharmacogn. Res. 2011, 3, 100-106. [CrossRef]

40. Ku, W.-F.; Tan, S.-J.; Low, Y.-Y.; Komiyama, K.; Kam, T.-S. Angustilobine and andranginine type indole alkaloids and an uleine-secovallesamine bisindole alkaloid from Alstonia angustiloba. Phytochemistry 2011, 72, 2212-2218. [CrossRef]

41. Marie-Magdeleine, C.; Udino, L.; Philibert, L.; Bocage, B.; Archimede, H. In vitro effects of Cassava (Manihot esculenta) leaf extracts on four development stages of Haemonchus Contortus. Vet. Parasitol. 2010, 173, 85-92. [CrossRef]

42. Yusuf, U.F.; Okechukwu, P.N. Anti-inflammatory, analgesic and anti-Pyretic activity of cassava leaves extract. Asia J. Pharm. Clin. Res. 2013, 6, 89-92.

43. Mbahi, M.A.; Mbahi, A.M.; Umar, I.A.; Ameh, D.A.; Joseph, I.; Amos, P.I. Phytochemical screening and antimicrobial activity of the pulp extract and fractions of Ziziphus mauritiana. Biochem. Anal. Biochem. 2018, 7, 1-6. [CrossRef]

44. Dutta, R.P.; Patil, M.B. Therapeutic potential of root and stem bark of wild medicinal plant Ziziphus mauritiana (Lamk.) against silica induced toxicity in Wistar albino rats. J. Ethnopharmacol. 2018, 224, 111-118. [CrossRef]

45. Verbeke, P.; Fonager, J.; Clark, B.F.C.; Rattan, S.I.S. Heat shock response and ageing: Mechanisms and applications. Cell Biol. Int. 2001, 25, 845-857. [CrossRef] 
46. Birben, E.; Sahiner, U.M.; Sackesen, C.; Erzurum, S.; Kalayci, O. Oxidative stress and antioxidant defense. World Allergy Organ. J. 2012, 5, 9-19. [CrossRef]

47. Wei, T.; Chen, C.; Hou, J.; Xin, W.; Mori, A. Nitric oxide induces oxidative stress and apoptosis in neuronal cells. Biochim. Biophys. Acta Mol. Cell Res. 2000, 1498, 72-79. [CrossRef]

48. Anderson, A.S.; Mirian, A.I.S.; Rodrigo, M.F.; Mariana, A.B.; Tamara, R.M.; Mariene, H.D.; Claudia, M.D.S.; Juliana, M.F.; Angelita, D.C. Antioxidants and chlorophyll in cassava leaves at three plant ages. Afr. J. Agric. Res. 2013, 8, 3724-3730. [CrossRef]

49. Stahl, W.; Sies, H. Antioxidant activity of carotenoids. Mol. Aspects Med. 2003, 24, 345-351. [CrossRef]

50. Young, A.J.; Lowe, G.L. Carotenoids-Antioxidant properties. Antioxidants 2018, 7, 28. [CrossRef]

51. Abu Bakar, M.F.; Abdul Karim, F.; Perisamy, E. Comparison of phytochemicals and antioxidant properties of different fruit parts of selected Artocarpus species from Sabah, Malaysia. Sains Malays. 2015, 44, 355-363. [CrossRef]

52. Abdelhakim, I.A.; Abdel-baky, A.M.; Bishay, D.W. In vitro evaluation of antioxidant activity of Caryota mitis Lour. Leaves extracts. J. Pharmacogn. Phytochem. 2017, 6, 2559-2562.

53. Cao, G.; Sofic, E.; Prior, R.L. Antioxidant and prooxidant behavior of flavonoids: Structure-activity relationships. Free Radic. Biol. Med. 1997, 22, 749-760. [CrossRef]

54. Wang, M.; Meng, D.; Zhang, P.; Wang, X.; Du, G.; Brennan, C.; Li, S.; Ho, C.-T.; Zhao, H. Antioxidant protection of nobiletin, 5-demethylnobiletin, tangeretin, and 5-demethyltangeretin from citrus peel in Saccharomyces Cerevisiae. J. Agric. Food Chem. 2018, 66, 3155-3160. [CrossRef] [PubMed] 